\title{
ESTUDO PRELIMINAR DA PREFERÊNCIA DE COR PARA ROTA E SETA DE MANOBRA EM MAPAS DE SISTEMAS DE GUIA DE ROTA
}

Color preference for route and direction arrow in maps of route guidance systems

\author{
ANA PAULA MARQUES RAMOS ${ }^{1}$ \\ EDMUR AZEVEDO PUGLIESI ${ }^{2}$ \\ MÔNICA MODESTA SANTOS DECANINI ${ }^{3}$ \\ VILMA MAYUMI TACHIBANA ${ }^{4}$ \\ Universidade Estadual Paulista - UNESP \\ ${ }^{1}$ Programa de Pós-Graduação em Ciências Cartográficas \\ ${ }^{2,3}$ Departamento de Cartografia \\ ${ }^{4}$ Departamento de Estatística \\ Rua Roberto Simonsen, 305 \\ CEP 19060-900 - Presidente Prudente - São Paulo \\ marques.engcart@gmail.com; \{edmur; monca; vilma $\} @$ fct.unesp.br $\}$
}

\begin{abstract}
RESUMO
Os Sistemas de Navegação e Guia de Rota em Automóvel (SINGRA), comerciais ou gratuitos, vêm adotando diferentes projetos gráficos para os mapas que apoiam a tarefa de navegação. O objetivo deste trabalho é avaliar a preferência de um grupo de motoristas pela cor da rota e da seta de manobra para mapas destes sistemas. Um total de 54 motoristas ( 27 homens e 27 mulheres) participou de um experimento que ocorreu em um automóvel estacionado. Duas representações cartográficas foram produzidas para avaliar a preferência pela cor da rota, e outras duas representações para avaliar a preferência pela cor da seta de manobra. Para a cor da rota, utilizou-se o preto e o matiz azul, e para a cor da seta, utilizou-se os matizes verde e vermelho. As representações foram exibidas em um monitor de navegação sete polegadas. Os resultados indicam que a maioria dos motoristas prefere a rota em preto. Porém, os motoristas experientes com SINGRA apresentam tendência para a rota no matiz azul. A seta em verde foi preferida pela grande maioria dos motoristas e nenhuma relação foi encontrada com as características individuais. Neste estudo, conclui-se
\end{abstract}


que a preferência dos motoristas foi influenciada por diferentes fatores, tais como agrupamento perceptivo por cor, forma e proximidade, associação subjetiva ou convencional e pelo nível de contraste entre as feições do mapa. Recomenda-se avaliar o desempenho das representações cartográficas na tarefa de manutenção em rota para corroborar a influência do projeto gráfico dos mapas na usabilidade dos sistemas de guia de rota.

Palavras-chave: Sistema de Navegação e Guia de Rota; Representação Cartográfica; Preferência Subjetiva; Usabilidade.

\section{ABSTRACT}

In-Car Navigation System and Route Guidance (RGNS), commercial or free, have adopted different graphic designs to maps which support the navigation task. This paper aims at evaluating the driver's subjective preference for colors to represent route and arrow direction in maps for these systems. A total of 54 drivers (27 males and 27 females) participated in an experiment which was performed in a parked vehicle. Two cartographic representations were produced to evaluate the route color and other two for the arrow color. The route was symbolized by black or blue color and the arrow direction by green or red hue. The cartographic representations were presented in a seven-inch navigation monitor. The results indicate that most drivers prefer the navigation route in black color. However, drivers who regularly use navigation systems have presented the tendency to prefer the route in blue hue. The direction arrow in green hue was the mostly preferred by the drivers and there was no significant correlation between different individual characteristics. This study concludes that drivers' preference was influenced by different factors, such as grouping by color, shape and proximity, subjective or conventional association, as well as by the level of contrast among the map features. Evaluating the performance of cartographic representations in route following task is recommended, in order to support the influence of graphic designs to maps in usability of route guidance systems.

Keywords: Route Guidance and Navigation System; Cartographic Representation; Subjective Preference; Usability.

\section{INTRODUÇÃO}

Os esquemas de seta (turn-by-turn) são as primeiras modalidades visuais utilizadas pelos Sistemas de Navegação e Guia de Rota em Automóvel (SINGRA) para auxiliar o motorista na tarefa de navegação, e nestas modalidades são indicados tanto a direção da manobra quanto o prosseguimento na rota (DINGUS; HULSE, 1993; OBATA; DAIMON; KAWASHIMA, 1993; PUGLIESI; DECANINI, 2009a). A partir do final do século XX, a evolução tecnológica possibilitou o incremento de novas informações às representações cartográficas de apoio à navegação. A indicação da posição do automóvel na rota e apresentação de pontos de referência são alguns exemplos de informações adicionadas aos mapas (LABIALE, 2001;

Bol. Ciênc. Geod., sec. Artigos, Curitiba, v. 20, nº 2, p.317-333, abr-jun, 2014. 
HWAN; JIN, 2010; PUGLIESI; DECANINI, 2009b). Dentre os símbolos acrescentados às representações cartográficas e de maior relevância ao motorista destacam-se a rota de navegação e a seta de indicação da direção da manobra (MAY; ROSS; BAYER, 2003; WU; ZHANG, 2009).

$\mathrm{O}$ símbolo correspondente à rota é utilizado para descrever o trajeto entre a origem e o destino de interesse. Uma das finalidades de se destacar a rota sobre a malha viária é facilitar a percepção do trajeto em desenvolvimento para auxiliar o motorista no estágio de 'confirmação da viagem' (BURNETT, 1998); um estágio importante para manter o motorista seguro e confiante no trajeto (LEE; FORLIZZI; HUDSON, 2008). O realce da rota no mapa tem sido realizado a partir da criação de uma hierarquia visual entre os elementos lineares por meio do uso das variáveis visuais cor, tamanho e/ou forma (HO; LI, 2004; PUGLIESI et al., 2004; MARQUES; DECANINI; PUGLIESI, 2012). Quanto ao símbolo correspondente à seta de manobra, este tem a função de enfatizar a localização e a direção da manobra (BURNETT, 1998; LABIALE, 2001; PUGLIESI; DECANINI; TACHIBANA, 2009). A seta consiste em um símbolo importante para auxiliar na tarefa tática, a qual está relacionada à etapa de preparação para realizar uma manobra (MICHON, 1985; PUGLIEISI et al., 2013); por isso, consiste em uma tarefa que demanda maior carga mental de trabalho do motorista (BURNETT, 1998; KABER et al., 2012).

Independentemente do tipo de símbolo ou da quantidade de elementos exibido por um SINGRA, o projeto gráfico dos mapas para estes sistemas deveria ser cuidadosamente preparado para minimizar ou evitar ruídos no processo de comunicação cartográfica. Uma etapa importante no projeto gráfico refere-se à seleção da cor dos elementos (DENT; TORGUSON; HODLER 2009; MARTINELLI, 2011). Devido a sua alta capacidade seletiva para criação e separação de grupos distintos, a cor é uma das variáveis visuais mais importantes para a comunicação visual (DENT; TORGUSON; HODLER 2009; SLOCUM et al., 2009). Para os mapas de SINGRA, a seleção da cor torna-se especialmente complexa, pois estes sistemas são utilizados com o automóvel em movimento (LAVIE; ORON-GILAD; MEYER, 2011), o que demanda do motorista o processo de atenção dividida entre a via e o mapa (WICKENS et al., 2004).

Os símbolos, tais como o automóvel, a rota e a seta de manobra deveriam possuir uma característica visual única, não ambígua, de modo que permita a fácil e a rápida diferenciação dos outros elementos usados como contexto espacial na navegação. A falta de segregação ou de agrupamento visual entre os símbolos prejudica o processo de comunicação cartográfica e, com isso, pode reduzir o desempenho do mapa (DENT; TORGUSON; HODLER, 2009; SLOCUM et al., 2009). Ao analisar o projeto gráfico dos mapas de sistemas de navegação, gratuitos ou comerciais, nota-se que há uma diversidade de projetos gráficos. Para representar a rota, a cor-matiz azul, por exemplo, vêm sendo muito utilizada pelos sistemas. Entretanto, o uso deste matiz para a rota pode ser um problema para o desempenho dos mapas, pois o motorista pode ser induzido a realizar uma associação equivocada com cursos d'água. Para representar a seta, os sistemas têm utilizado cores diversas, 
sem que haja uma possível associação do referente com o significante. Neste sentido, a avaliação do projeto gráfico de mapas para SINGRA, tal como o estudo da cor apropriada para os símbolos de apoio à navegação, torna-se uma medida necessária quando se pretende contribuir com a usabilidade de sistemas desta natureza.

O objetivo deste trabalho é avaliar a preferência subjetiva dos motoristas pela cor da rota e seta de manobra em mapa de sistema de navegação e guia de rota. As questões colocadas são: "Qual é a cor preferida para representar a rota: preta ou azul?"; "Qual é a cor preferida para representar a seta de direção da manobra: verde ou vermelha?"; e "Há relação entre a preferência e as características individuais dos motoristas?". Com estas respostas, pretende-se fornecer indicações de projeto às indústrias que desenvolvem este tipo sistema visando auxiliar o motorista na tarefa de navegação em automóvel, a qual é complexa por demandar a execução simultânea de atividade cognitivo-motora.

\section{MÉTODO}

\subsection{Participantes}

Um total de 54 motoristas, com idade entre 22 e 40 anos, participou do teste de preferência subjetiva (Tabela 1). A seleção dos candidatos foi realizada com base em três critérios. Primeiro, possuir Carteira Nacional de Habilitação $(\mathrm{CNH})$ e ter experiência com a direção de automóvel por pelo menos dois anos, pois estas pessoas caracterizam-se como motoristas que dirigem automóvel com regularidade (BURNETT, 1998; PUGLIESI; DECANINI; TACHIBANA, 2009). Segundo, não apresentar disfunção na visão de cores (daltonismo), pois as cores dos símbolos podem não ser detectadas por pessoas daltônicas (MACEACHREN, 1995; RIGDEN, 1999). Terceiro, ter idade até 40 anos, pois os efeitos da idade prejudicam o processo de acomodação focal (SEKULER, SEKULER, 2000) e interferem no desempenho do motorista na direção (LIU, 2000). A partir dos 30 anos de idade, o olho humano perde gradativamente a sensibilidade ao contraste nos comprimentos de onda longo (ex.: vermelho) e médio (ex.: verde) (SEKULER; SEKULER, 2000).

Tabela 1 - Participantes do teste de preferência subjetiva.

\begin{tabular}{l|c|c|c}
\hline \multirow{2}{*}{ Faixa etária } & \multicolumn{2}{c|}{ Gênero } & \multirow{2}{*}{ Total } \\
\cline { 2 - 3 } & Homem & Mulher & \\
\hline A partir de 22 anos até menos de 30anos & 17 & 15 & 27 \\
\hline A partir de 30 anos até 40 anos & 10 & 12 & 27 \\
\hline Total & 27 & 27 & 54 \\
\hline Média e desvio-padrão & & 29 e 5,0 \\
\hline
\end{tabular}


Para apoiar a etapa de seleção dos candidatos e de investigação da associação entre as características individuais e a preferência dos motoristas elaborou-se um questionário de caracterização individual, o qual foi aplicado em forma de entrevista estruturada conforme recomenda Young e Stanton (2005). Dentre os dados coletados dos candidatos pode-se destacar: o gênero, a experiência com sistemas de navegação e guia de rota, a formação educacional, o tempo de habilitação e características oftalmológicas. A experiência com SINGRA foi coletada mediante a aplicação da seguinte pergunta: "Com que frequência você utiliza GPS de navegação para dirigir?”. As opções oferecidas foram: 'frequentemente' (uma ou mais vezes por semana); 'ocasionalmente' (no máximo uma vez por mês); 'raramente' (uma ou duas vezes por ano); ou 'nunca'.

Adotou-se como técnica de recrutamento dos candidatos a abordagem pessoal, na qual se realizou um esclarecimento breve sobre o objetivo da pesquisa. Ao mostrar interesse pelo teste, o candidato forneceu o dia e horário de maior disponibilidade para realizá-lo. Todos os motoristas participantes são residentes na cidade de Presidente Prudente/SP, local de realização do teste, e o grupo constituiuse basicamente de alunos, docentes e funcionários da Faculdade de Ciências e Tecnologia (FCT/UNESP).

\subsection{Aparato}

O teste foi realizado em um automóvel estacionado em via urbana, durante o período diurno para simular o uso de um SINGRA no ambiente de direção de um automóvel. Adotou-se o período diurno para a realização do teste, pois, de acordo com Pugliesi (2007), a preferência do motorista pelas cores dos símbolos dos mapas é influenciada pelo período no qual a navegação ocorre, isto é, se dia ou noite. Manteve-se ligado o motor e o ar condicionado do veículo, respectivamente, para aumentar o estado de atenção do motorista e proporcionar conforto durante a experimentação.

Um dispositivo visual do tipo tablet (Asus FonePad ME371MG), com tela de sete polegadas, foi utilizado como monitor de navegação para exibir as representações cartográficas aos motoristas. A mídia nesta dimensão vem sendo adotada com frequência para os sistemas comerciais (LI et al., 2012), bem como consiste no padrão adotado pelas montadoras de automóveis, tais como Hyundai, $B M W$ e Peugeot, para disponibilizar sistemas de guia de rota como item de série nos seus veículos. Manteve-se a mídia na resolução de vídeo de 1024 x 768 pixels.

A partir de um suporte veicular apropriado, fixou-se o monitor de navegação no para-brisa do automóvel, direcionando-o à face do motorista em um ângulo menor que $30^{\circ}$ entre o centro da via e o centro do monitor. Esta posição foi selecionada porque permite ao motorista realizar o processo de busca visual pelos elementos do mapa em menor tempo (BURNETT et al., 2012; WITTMANN et al., 2006). O tablet foi utilizado também para gravar as respostas fornecidas por meio da voz dos motoristas. A gravação das respostas facilitou a aplicação do teste e as 
etapas de organização dos dados e análise dos resultados. A Figura 1 mostra o cenário do teste.

Figura 1 - Cenário do teste.

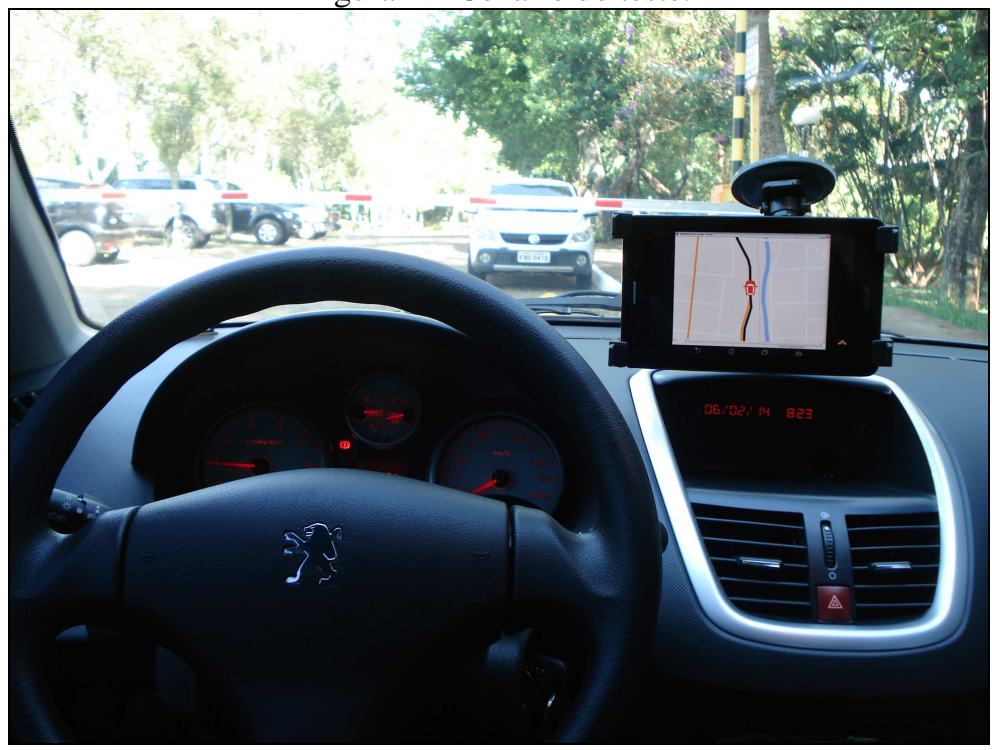

\subsection{Representações Cartográficas}

Um conjunto de representações cartográficas na escala 1/3.000 foi elaborado para apoiar o teste de preferência subjetiva. As representações foram projetadas no software ESRI ArcGIS e convertidas para o formato imagem a fim de serem exibidas no monitor de navegação usando o Microsoft Power Point. As representações cartográficas produzidas fazem referência a um trecho de rota na cidade de Álvares Machado/SP.

O projeto gráfico das representações baseia-se em um protótipo de SINGRA desenvolvido em trabalhos anteriores (PUGLIESI; DECANINI; TACHIBANA, 2009; MARQUES; DECANINI; PUGLIESI, 2012). Neste protótipo, os mapas são apresentados com esquema de referência egocêntrico e sistema de projeção cilíndrico ortogonal. As informações que compõem estes mapas são a rota de navegação, a seta de manobra, o símbolo do automóvel $(\mathrm{RGB}=255,0,0)$ e os elementos que fornecem contexto espacial na navegação, tais como ferrovia $(\mathrm{RGB}=230,0,220)$, vias principais $(\mathrm{RGB}=255,166,0)$ e vias locais $(\mathrm{RGB}=0,0,0)$. Tais classes de informação são as principais para apoiar a navegação em ambientes urbanos pouco ou não conhecidos pelos motoristas (BURNETT, 1998; MAY; ROSS; BAYER, 2003; ROSS et al., 1996). 
Em virtude do teste se concentrar na preferência subjetiva pela cor da rota e da seta de manobra, alterou-se somente a variável visual cor destes dois elementos. Um total de quatro representações foi preparado, sendo duas para representar a rota, e outras duas para representar a seta de manobra. Quanto à rota, o primeiro mapa (Figura 2a) a manteve em preto a fim de estabelecer uma associação com a cor do asfalto, conforme propõem Pugliesi, Decanini e Tachibana (2009). O segundo mapa representou a rota na cor-matiz azul por três razões. Primeiro, por esta cor ser adotada em dois importantes portais eletrônicos de localização e planejamento de rota disponível na internet, o Google Maps e o Maplink. Segundo, pela cor-matiz ser adotada em diversos mapas de SINGRA, gratuitos, tais como o Google Maps Navigation (sistema da Google), ou comerciais, tais como Multilaser, Destinator, Medion, Mio, NDrive. Terceiro, pela cor-matiz azul ser utilizada de maneira associativa para representar feições hidrográficas (DENT; TORGUSON; HODLER 2009). Devido os símbolos da rota e dos cursos d'águas desenvolverem, respectivamente, a relação de figura e fundo nos mapas de sistemas de guia de rota, tem-se que a associação entre estes elementos pode afetar negativamente a legibilidade da representação e reduzir o desempenho da comunicação cartográfica. A cor é uma variável visual que fortalece o agrupamento perceptivo por similaridade e proximidade (DENT; TORGUSON; HODLER 2009; GOMES FILHO, 2002). Sendo assim, para identificar se a preferência pela cor da rota é influenciada pela proximidade de um curso d'água, inseriu-se um trecho de curso d'água fictício nas representações produzidas no ArcGIS. O matiz azul adotado é similar ao utilizado nos mapas do Google Maps ( $\mathrm{RGB}=148,148,255)$ (Figura 2b).

Quanto às cores selecionadas para a seta de manobra, optou-se por dois matizes, o verde $(\mathrm{RGB}=76,230,0)$ e o vermelho $(\mathrm{RGB}=255,0,0)$. O propósito da seta na cor-matiz verde foi manter a similaridade com a cor adotada para as setas de manobra presentes no painel dos automóveis. Além disso, teve-se o intuito de manter uma associação com a luz verde dos semáforos, a qual comunica ao motorista a mensagem de seguir em frente. Vale ressaltar que este matiz é adotado em diversos mapas de sistemas comercias, tais como NDrive, Mio, Dynamic e Multilaser. O segundo mapa relacionado à seta manteve a proposta de Pugliesi, Decanini e Tachibana (2009), na qual a seta de manobra é simbolizada no matiz vermelho para estabelecer um agrupamento perceptivo por cor com o automóvel. A criação de unidade perceptiva entre os elementos contribui para a rápida leitura de uma representação visual (GOMES FILHO, 2002). Por ser uma cor quente, o matiz vermelho avança aos olhos (DENT; TORGUSON; HODLER 2009) e é indicado para representar elementos de pequena dimensão, de acordo com o mapa de sensibilidade retinal (WADE; SWANSTON, 1991 apud MACEACHREN, 1995). A Figura 3 apresenta as representações da seta. 
Figura 2 - Mapa com rota em preto (a) e em azul (b).

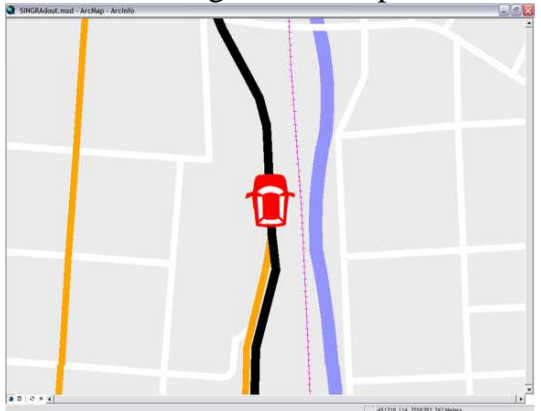

(a)

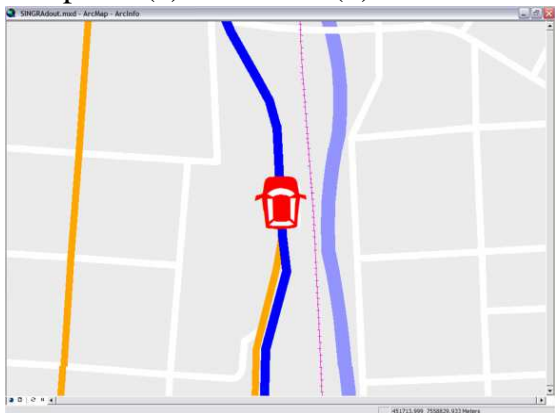

(b)

Figura 3 - Mapa com seta em verde (a) e vermelho (b).

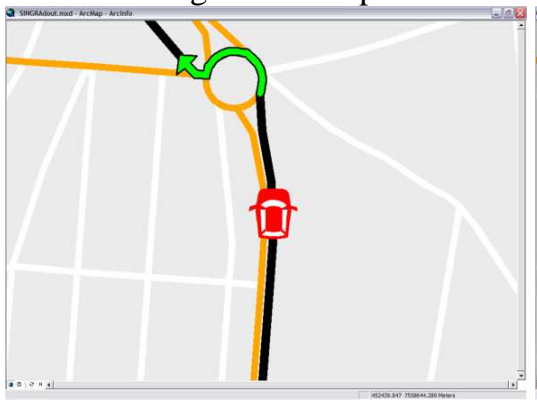

(a)

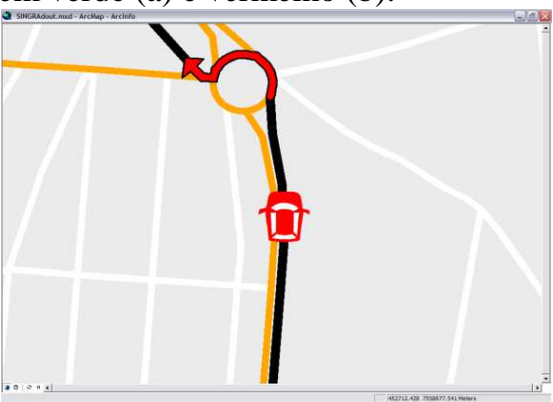

(b)

\subsection{Procedimento}

O teste foi iniciado com a entrada do motorista no automóvel e sua preparação como se fosse dirigir o veículo. A próxima etapa consistiu na apresentação do roteiro do teste, o qual foi lido em voz alta pelo experimentador, para explanar o objetivo da pesquisa e sugerir que o motorista compreendesse o ambiente como se estivesse dirigindo o automóvel com o apoio de um SINGRA. Na sequência, o motorista leu e assinou o termo de consentimento livre e esclarecido para registrar sua aceitação formal em participar do teste, bem como preencheu o questionário de caracterização individual. Em seguida, o experimentador apresentou o manual de símbolos do SINGRA para familiarizar o motorista quanto às representações cartográficas que seriam exibidas na tela do monitor de navegação. Como mostra a Figura 4, o manual de símbolos do SINGRA utilizou cores para a rota e para a seta diferentes daquelas avaliadas. Posteriormente, o experimentador aplicou a primeira pergunta do questionário de preferência subjetiva, a qual consistiu em: "Qual cor de rota você prefere: PRETA ou AZUL?”. A partir desta questão, cada representação cartográfica foi exibida no por um tempo mínimo de cinco segundos na tela do monitor de navegação e, em seguida, fez-se a pergunta "Por quê?". Na sequência, 
aplicou-se a segunda pergunta do teste: "Qual cor de seta você prefere para indicar a direção da manobra: VERDE ou VERMELHA?", seguida da pergunta "Por quê?". Os dados foram armazenados pelo tablet, na forma de arquivos de áudio, e pelo experimentador, por meio de anotações. A duração média do procedimento de teste com cada motorista foi inferior a 20 minutos e independente de qualquer relação com o aspecto perceptivo.

Figura 4 - Manual de símbolos do SINGRA para exemplificar a rota (a) e a seta de manobra (b).



(a)

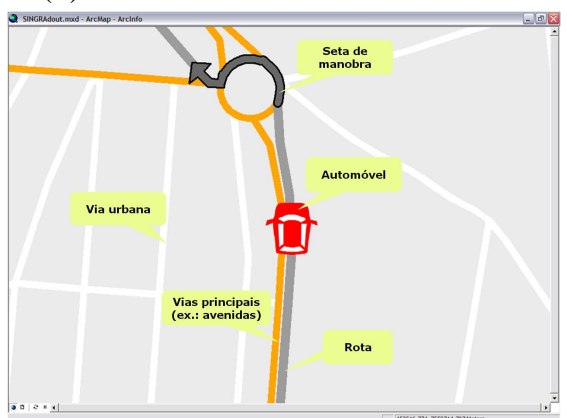

(b)

\subsection{Análises Estatísticas}

Para processar os dados, utilizou-se o software SPSS 16.0 (Statistical Package for the Social Sciences) disponível na FCT/UNESP. A ocorrência de diferenças estatisticamente significativas na preferência dos motoristas foi identificada a partir de análise não-paramétrica. Este tipo de análise é indicado para dados de natureza qualitativa, bem como quando não se conhece a distribuição de probabilidade que possuem (SIEGEL, 1975; CONOVER, 1999). Os testes foram aplicados para a Frequência Observada $\left(\mathrm{F}_{0}\right)$ em cada grupo avaliado. Os grupos com $\mathrm{F}_{0}$ inferior a cinco elementos foram avaliados pelas provas Binomial (caso de uma amostra) e Fisher (caso de duas amostras). Enquanto os grupos com $\mathrm{F}_{0}$ igual ou superior a cinco elementos foram avaliados pelo método Qui-Quadrado $\left(\chi^{2}\right)$, caso de uma ou duas amostras não relacionadas. Em todas as análises, fixou-se o nível de significância em $5 \%$.

\section{RESULTADOS E DISCUSSÕES}

\subsection{Preferência Subjetiva}

Duas análises foram realizadas. A primeira análise identifica a preferência pelo matiz que representa a rota de navegação. Os resultados revelam que $78 \%$ dos motoristas preferem a rota em preto e apenas $22 \%$ preferem a rota em azul. O teste Qui-Quadrado mostra $\left(\chi^{2}=16,66, \mathrm{p}<0,0001\right)$ que esta diferença é estatisticamente 
significativa, permitindo afirmar que a maioria dos motoristas prefere visualizar a rota de navegação em preto nos mapas de sistema de guia de rota.

Para compreender a razão pela preferência da rota em preto, fez-se uma interpretação das justificativas dos motoristas. Os comentários que apareceram com maior frequência encontram-se na Tabela 2 , a partir da qual se nota que a representação da rota e do curso d'água no mesmo matiz dificulta a rápida identificação do trajeto estabelecido.

Tabela 2 - Comentários dos motoristas sobre a cor da rota.

\begin{tabular}{c|l|l}
\hline Rota & \multicolumn{1}{|c|}{ Comentários } & \multicolumn{1}{|l}{ Interpretação dos comentários } \\
\hline \multirow{3}{*}{ Preta } & $\begin{array}{l}\text { "se associa com a cor do } \\
\text { asfalto". }\end{array}$ & $\begin{array}{l}\text { Favorece a associação do } \\
\text { referente com o significante. }\end{array}$ \\
\cline { 2 - 3 } & $\begin{array}{l}\text { "não confunde com a cor do "mais realçada no } \\
\text { mapa". }\end{array}$ & $\begin{array}{l}\text { Favorece a segregação } \\
\text { de figura e fundo. }\end{array}$ \\
\hline \multirow{2}{*}{ Azul } & "lembra um rio". & $\begin{array}{l}\text { Relação equivocada entre } \\
\text { referente e significante. }\end{array}$ \\
\cline { 2 - 3 } & "pouco destaque no mapa". & Prejudica a relação figura-fundo \\
\hline
\end{tabular}

A dificuldade de se realizar a rápida discriminação entre a rota e o curso d'água é explicada em função de três tipos de agrupamento que ocorre entre estas feições. Primeiro, o agrupamento perceptivo por cor. Os matizes similares, ou com apenas variação de brilho, têm a tendência de se agruparem (DENT; TORGUSON; HODLER, 2009). Segundo, o agrupamento perceptivo por similaridade. Tanto a rota quanto o curso d'água são representados pela primitiva de linha; portanto, ambas possuem forma linear semelhante. Gomes Filho (2002) afirma que os elementos com características geométrica similares, tal como forma, tamanho, orientação, tendem a formar grupos. Terceiro, o agrupamento perceptivo por proximidade (GOMES FILHO, 2002). Pela Figura 2, nota-se que a rota está localizada próxima a um curso d'água, favorecendo o agrupamento entre tais elementos. Nesse sentido, enquanto a rota em azul se associa às feições hidrográficas e prejudica a segregação de figura e fundo, a rota em preto, além de aumentar o contraste do mapa, também permite uma associação com a representação do asfalto, fortalecendo a relação entre referente (elemento do mundo real) e significante (elemento representado no mapa).

Nos casos estudados, ao representar a rota no matiz azul, os agrupamentos por similaridade e proximidade foram fortalecidos pelo agrupamento por cor e isto desfavoreceu a legibilidade do mapa, uma vez que o intuito é o de segregar a rota do curso d'água e não unificar tais feições. Em representações cartográficas para sistemas de guia de rota, a rota de navegação e o curso d'água devem consistir, respectivamente, em figura e fundo. Assim, a criação de uma hierarquia visual entre tais feições é fundamental para instruir o motorista enquanto divide a atenção entre 
a via e o sistema. A falta de segregação entre os símbolos do mapa implica em ruídos no processo de comunicação cartográfica (DENT; TORGUSON; HODLER, 2009; SLOCUM et al., 2009). No contexto de SINGRA, tais ruídos podem resultar no aumento da demanda visual do motorista, o que significa mais tempo com os olhos fora da via e, por conseguinte, riscos à segurança no trânsito.

A segunda análise identifica a preferência pelo matiz que representa a seta de manobra. Os resultados revelam que $93 \%$ dos motoristas preferem a seta no matiz verde e apenas $7 \%$ preferem a seta no matiz vermelho. A significância desta discrepância é confirmada pelo teste Binomial ( $p<0,0001)$, permitindo afirmar que os usuários de sistema e guia de rota preferem visualizar a seta de manobra no matiz verde comparado ao matiz vermelho. A principal justificativa dos motoristas pela preferência da seta em verde é por se destacar no mapa, realçando a direção da manobra (Tabela 3).

Tabela 3 - Comentários dos motoristas sobre a cor da seta.

\begin{tabular}{|c|c|c|}
\hline Rota & Comentários & $\begin{array}{l}\text { Interpretação dos } \\
\text { comentários }\end{array}$ \\
\hline \multirow{3}{*}{ Verde } & $\begin{array}{l}\text { "se destaca mais em relação } \\
\text { à rota em preto e as vias } \\
\text { principais em laranja". }\end{array}$ & $\begin{array}{l}\text { Favorece a segregação de } \\
\text { figura e fundo. }\end{array}$ \\
\hline & $\begin{array}{l}\text { "se associa à luz verde dos } \\
\text { semáforos". }\end{array}$ & $\begin{array}{l}\text { Favorece a associação } \\
\text { com sinais de trânsito } \\
\text { pré-estabelecidos. }\end{array}$ \\
\hline & $\begin{array}{l}\text { "se associa à instrução } \\
\text { 'passagem livre', 'prossiga', } \\
\text { 'siga em frente"”. }\end{array}$ & $\begin{array}{l}\text { Favorece a associação com } \\
\text { diversos tipos de sinais de } \\
\text { natureza similar. }\end{array}$ \\
\hline \multirow[t]{2}{*}{ Vermelha } & $\begin{array}{l}\text { "se associa à instrução } \\
\text { 'pare', 'proibido', 'perigo'”. }\end{array}$ & $\begin{array}{l}\text { Favorece a associação com } \\
\text { diversos tipos de sinais de } \\
\text { natureza similar. }\end{array}$ \\
\hline & $\begin{array}{l}\text { "confunde com a cor do } \\
\text { automóvel". }\end{array}$ & $\begin{array}{l}\text { Desfavorece a segregação } \\
\text { entre seta e automóvel }\end{array}$ \\
\hline
\end{tabular}

Este realce da manobra contribuiu positivamente para a legibilidade da representação cartográfica e é explicado pelo processo de contraste simultâneo. $\mathrm{O}$ princípio do contraste simultâneo é que nenhuma cor tem valor por si só, ou seja, diferentes cores justapostas exercem influência recíproca entre si (DENT; TORGUSON; HODLER, 2009). Neste caso, um mesmo matiz pode ser acentuado, atenuado ou modificado de acordo com as cores superpostas (DENT; TORGUSON; HODLER, 2009; PEDROSA, 2009; SLOCUM et al., 2009). Com base nesta definição e na análise visual da Figura 3, observa-se um maior contraste entre a seta no matiz verde e a rota em preto e as vias principais no matiz laranja (Figura 3a) comparado à simbolização da seta no matiz vermelho (Figura 3b). A seta em 
vermelho é atenuada pela presença do matiz laranja nas vias principais. Por outro lado, o matiz laranja acentua a seta em verde. Pelo sistema aditivo de cores, isto ocorre porque, embora o laranja seja uma combinação dos matizes verde e vermelho, há uma maior concentração de vermelho na composição desta cor secundária comparado à quantidade de matiz verde (PEDROSA, 2009), diminuindo o contraste entre as feições em laranja e vermelho. O baixo contraste entre a seta em vermelho e as vias principais em laranja deve-se ainda ao fato destes matizes serem cores quentes; portanto, agrupam-se. As cores frias (ex.: verde, azul) e as cores quentes (ex.: vermelho e laranja) têm a tendência de se agruparem e, com isso, de reduzirem o contraste entre as feições que simbolizam (DENT; TORGUSON; HODLER, 2009). A combinação de cores quentes ou frias deve ser evitada quando o objetivo é estabelecer a segregação entre os elementos do mapa (GOMES FILHO, 2002; PEDROSA, 2011).

Outro aspecto que contribuiu para o realce da manobra ao utilizar a seta no matiz verde é a ocorrência de maior contraste entre este matiz e o preto da rota e do contorno da seta comparado ao matiz vermelho. A percepção humana, por cor, forma ou tamanho não é absoluta, mas ocorre por relações (GOMES FILHO, 2002). Assim, o preto presente na rota e no contorno da seta produziu a sensação perceptiva de um matiz verde com mais brilho comparado ao matiz vermelho, ainda que ambos possuam o mesmo nível de saturação. O contraste entre a rota em preto, as vias principais em laranja e a seta em verde aumentou a legibilidade do mapa, pois realçou a relação de figura-fundo na representação. Este ganho de legibilidade no mapa pode auxiliar o motorista na realização da tarefa tática, reduzindo o número de erros de manobra, visto que nesta tarefa o motorista identifica e discrimina a direção que deve ser tomada na junção.

Uma segunda justificativa de grande parte dos motoristas é o fato do matiz verde da seta se associar à luz verde dos semáforos. Como mostra a Tabela 3 , a seta em verde comunica a informação 'siga em frente' ou de que a manobra a ser realizada está correta. Enquanto a seta em vermelho transmite a mensagem de que é proibido virar naquela via ou de que a manobra está incorreta. Por fim, a maioria dos motoristas argumentou que a representação da seta e do carro no mesmo matiz é um problema, porque dificulta a rápida percepção da direção da manobra devido à necessidade de dividir a atenção entre estes dois símbolos. Nos casos estudados, este processo de atenção dividida consiste em um ruído na comunicação da informação. Devido o sistema humano de processamento da informação possuir capacidade limitada (PUGLIESI et al., 2013; WICKENS, et al., 2004), os mapas para SINGRA devem requerer o mínimo de processamento cognitivo do motorista, pois sua tarefa principal é dirigir com segurança.

\subsection{Características Individuais}

Para verificar se há associação entre as características individuais dos motoristas e a preferência pela cor da rota e da seta de manobra, os dados de 
caracterização individual foram organizados em três distintas classes qualitativas em termos das frequências observadas em cada classe conforme indicado na Tabela 4.

Tabela 4 - Características individuais dos participantes do teste.

\begin{tabular}{l|c|c|c}
\hline \multirow{2}{*}{ Gênero } & Homem & Mulher & Total \\
\cline { 2 - 4 } $\begin{array}{l}\text { Formação } \\
\text { Educacional }\end{array}$ & 27 & 27 & 54 \\
\cline { 2 - 4 } $\begin{array}{l}\text { Experiência } \\
\text { com SINGRA }\end{array}$ & $\begin{array}{c}\text { Pelo menos uma } \\
\text { vez por semana }\end{array}$ & $\begin{array}{c}\text { Cutegoria B } \\
\text { duas ou no máximo por ano }\end{array}$ & Total \\
\cline { 2 - 4 } & 21 & 33 & 54 \\
\hline
\end{tabular}

$\mathrm{Na}$ classe 'Formação Educacional', o grupo pertencente à categoria 'A' compreende sujeitos habilitados a produzir e interpretar mapas, tais como engenheiro cartógrafo, engenheiro ambiental, geógrafo, geólogo ou arquitetos. O grupo pertencente à categoria ' $\mathrm{B}$ ' compreende os sujeitos sem esta habilitação profissional ou com formação apenas de nível médio. Na classe 'experiência com SINGRA', optou-se por agrupar as opções 'frequentemente e ocasionalmente', bem como 'raramente e nunca' nas respectivas subclasses 'Pelo menos uma vez por semana' e 'Nunca ou no máximo duas vezes por ano'.

Os resultados do teste Qui-Quadrado (Tabela 5) mostram que o gênero do motorista não influenciou na preferência pela cor da rota ou da seta. A proporção de homens que preferem a rota em preto e a seta de manobra em verde é similar à proporção de mulheres que relatam esta mesma preferência.

Tabela 5- Preferência subjetiva e características individuais.

\begin{tabular}{|c|c|c|c|c|}
\hline \multirow{2}{*}{ Características individuais } & \multicolumn{2}{|c|}{ Rota } & \multicolumn{2}{|c|}{ Seta } \\
\hline & Preta & Azul & Verde & Vermelha \\
\hline Gênero: & & & & \\
\hline Homem & $74 \%$ & $26 \%$ & $93 \%$ & $7 \%$ \\
\hline Mulher & $81,5 \%$ & $18,5 \%$ & $93 \%$ & $7 \%$ \\
\hline Teste & \multicolumn{2}{|c|}{$\chi^{2}=0,429 \mathrm{p}=0,513$} & \multicolumn{2}{|c|}{ Fisher $<0,001 \mathrm{p}=1,00$} \\
\hline Formação educacional: & & & & \\
\hline Categoria A & $80 \%$ & $20 \%$ & $95 \%$ & $5 \%$ \\
\hline Categoria B & $76,5 \%$ & $23,5 \%$ & $91 \%$ & $9 \%$ \\
\hline Teste & \multicolumn{2}{|c|}{$\begin{array}{c}\text { Fisher }<0,001 \\
p=1,00\end{array}$} & \multicolumn{2}{|c|}{$\begin{array}{c}\text { Fisher }<0,001 \\
p=1,00\end{array}$} \\
\hline Experiência com SINGRA: & & & & \\
\hline Pelo menos $1 \mathrm{x}$ por semana & $62 \%$ & $38 \%$ & $90,5 \%$ & $9,5 \%$ \\
\hline Nunca ou no máximo 2x/ano & $88 \%$ & $12 \%$ & $94 \%$ & $6 \%$ \\
\hline Teste & \multicolumn{2}{|c|}{$\begin{array}{c}\text { Fisher }<0,001 \\
p=0,042\end{array}$} & \multicolumn{2}{|c|}{$\begin{array}{c}\text { Fisher }<0,001 \\
p=0,638\end{array}$} \\
\hline
\end{tabular}


As justificativas apresentadas pelos motoristas de ambos os gêneros se resumem às apresentadas nas Tabelas 1 e 2. Cabe destacar que apenas um motorista justificou a preferência pela seta em vermelho por esta criar uma unidade perceptiva com o símbolo do veículo em vermelho. Vale ressaltar que nenhuma associação significativa foi encontrada entre a preferência subjetiva e a faixa etária dos motoristas, ou seja, não houve diferença na preferência dos tons de cor para a rota $\left(\chi^{2}=0,459 \mathrm{p}=0,517\right)$ e para a seta (Fisher $\left.<0,001 \mathrm{p}=1,00\right)$ entre os motoristas com mais de 30 anos e os motoristas com menos de 30 anos de idade.

Quanto à característica individual 'Formação Educacional', os resultados revelam que preferência dos motoristas, tanto relacionada à cor da rota quanto à cor da seta (Tabela 5), independe da 'Formação Educacional' que estes possuem. Nos casos estudados, os motoristas habilitados a produzir e interpretar mapas têm a mesma preferência que os motoristas sem esta habilidade. Para a classe 'Experiência com SINGRA', identificou-se que motoristas que utilizam sistemas de guia de rota 'Pelo menos uma vez por semana' preferem a rota no matiz azul; enquanto os motoristas que utilizam tais sistemas no máximo duas vezes por ano preferem a rota em preto $(\mathrm{p}=0,042)$. Esta diferença pode ser explicada em função da representação da rota em preto ser pouco observada nos mapas de sistemas comercias ou gratuitos, diferentemente do matiz azul. Isto foi confirmado pelos relatos dos motoristas que preferiram a rota de navegação neste matiz. A associação subjetiva ou convencional (DENT; TORGUSON; HODLER, 2009) foi um fator que influenciou a preferência dos motoristas experientes com SINGRA. Quanto à seta de manobra, ambos os grupos de motoristas, experientes ou não com SINGRA, preferem a seta em verde.

\section{CONCLUSÕES E RECOMENDAÇÕES}

Este trabalho investigou a preferência dos motoristas pela cor da rota e da seta de manobra em mapas de Sistema de Navegação e Guia de Rota em Automóvel. Além disso, a investigação buscou compreender se há relação entre a preferência e as características individuais dos motoristas.

Conclui-se que os motoristas preferem visualizar a rota de navegação em preto comparada à cor-matiz azul. Este resultado decorreu do baixo contraste obtido entre a rota e o curso d'água quando ambas são representadas no mesmo matiz. Além disso, o agrupamento por cor fortaleceu o agrupamento por proximidade e similaridade, influenciando negativamente na relação de figura-fundo entre estas feições. Assim, recomenda-se que, devido o matiz azul ser associado tanto de maneira convencional quanto natural às feições hidrográficas, a rota de navegação em mapas de SINGRA seja representada por um matiz ou cor-valor diferente deste. Com isso, pode-se evitar ou minimizar as incertezas do motorista durante o processo reconhecimento da rota no mapa. Embora os motoristas experientes com SINGRA tenham tendência em preferir a rota no matiz azul, esta preferência foi atribuída ao fato dos sistemas não adotarem com frequência o preto para a rota. Sendo assim, sugere-se que futuros estudos sejam feitos apenas com motoristas experientes com SINGRA para verificar se a preferência pela rota no matiz azul se mantém em

Bol. Ciênc. Geod., sec. Artigos, Curitiba, v. 20, nº 2, p.317-333, abr-jun, 2014. 
relação ao preto.

Quanto à seta de manobra, conclui-se que a preferência dos motoristas se concentra no matiz que proporciona maior realce para a manobra. Estes resultados confirmam que a rápida e a clara identificação da direção da manobra é uma das metas do motorista na tarefa tática, as quais foram favorecidas pela representação da seta no matiz verde. Além de favorecer a segregação de figura e fundo, aumentando a legibilidade do mapa, este matiz é considerado o mais funcional para a seta de manobra por se associar às luzes dos semáforos. A seta no matiz vermelho induziu o processo de atenção dividida com o símbolo do automóvel e isto foi evidenciado com a menor aceitação dos motoristas por este matiz. Assim, recomenda-se que para a seleção da cor da seta de manobra seja considerado tanto aspectos relacionados ao aumento de contrate do mapa quanto de associação com os elementos do mundo real, tais como a sinalização de trânsito.

Embora essa investigação confirme a importância de se incluir a preferência subjetiva como uma variável no projeto de interfaces para sistemas de guia de rota, recomenda-se associar a preferência subjetiva com outras medidas de avaliação de natureza quantitativa, tais como demanda visual e erro navegacional, de maneira que se possa mensurar a carga mental de trabalho que as representações cartográficas geram nos motoristas. Além disso, recomenda-se avaliar os mapas propostos neste trabalho no contexto real da navegação por ser uma tarefa de maior demanda perceptiva e cognitiva para o motorista. Finalmente, sugere-se avaliar a preferência quanto às cores dos demais elementos do mapa, tais como vias secundárias e vias principais, pois estão presentes em grande quantidade no mapa e podem influenciar na percepção de outros símbolos.

\section{AGRADECIMENTOS}

$\mathrm{O}$ primeiro autor desse trabalho agradece ao $\mathrm{CNPq}$ pelo apoio financeiro com a bolsa de doutorado.

\section{REFERÊNCIAS BIBLIOGRÁFICAS}

BURNETT, G. E. "Turn right at the King's Head": Drivers' requirements for route guidance information. PhD Thesis, Loughborough University, UK. 1998.

BURNETT, G. E.; LAWSON, G.; MILLEN, L.; PICKERING, C.; WEBBER, E. Designing touchpad user-interfaces for right-hand drive vehicles: an investigation into where the touchpad should be located. Behaviour \& Information Technology. p.1-14. 2012.

CONOVER, W. J. Practical nonparametric statistics. New York: J. Wiley, 1999.

DENT, B. D.; TORGUSON, J.; HODLER, T. Cartography: Thematic Map Design. 6nd ed. Boston: McGraw Hill Higher Education. 2009.

DINGUS, T. A.; HULSE, M. C. Some human factors design issues and Recommendations for automobile navigation Information systems. Transportation Research Part C, v. 1, n. 2, p. 119-131. 1993. 
GOMES FILHO, J. Gestalt do Objeto: Sistema de Leitura Visual da Forma. São Paulo: Escrituras Editora. 2002.

HO, A.; LI, Z. Design of Dynamic Map for Land Vehicle Navigation. The Cartographic Journal. v. 41, n. 3, p. 265-270. 2004.

HWAN, L. J.; JIN, J. S. Design Guidelines and Recommendations for In-Vehicle Navigation Systems. Journal of Korean Society of Design Science. Korea. v. 23. n 3. p.309-327. 2010.

KABER, D. B.; LIANG, Y.; ZHANG, Y.; ROGERS, M. L.; GANGAKHEDKAR, S. Driver performance effects of simultaneous visual and cognitive distraction and adaptation behavior. Transportation Research Part F. Elsevier. v. 15, p.491-501. 2012.

LABIALE, G. Visual search and preferences concerning different types of guidance displays. Behaviour \& Information Technology. London: Taylor \& Francis. n. 3, v. 20, p.149-158. 2001.

LAVIE, T.; ORON-GILAD, T.; MEYER, J. Aesthetics and usability of in-vehicle navigation displays. International Journal of Human-Computer Studies. v. 69, p. 80-99. 2011.

LEE, J.; FORLIZZI, J.; HUDSON, S. Iterative design of MOVE: A situationally appropriate vehicle navigation system. International Journal of HumanComputer Studies. v. 66, p.198-215. 2008.

LI, B.; ZHU, K.; ZHANG, W.; WU, A.; ZHANG, X. A comparative study of two wayfinding aids for simulated driving tasks - single-scale and dual-scale GPS aids. Behaviour \& Information Technology. London:Taylor \& Francis. 2012.

LIU, Y. Effect of advanced traveler information system displays on younger and older drivers' performance. Displays. Elsevier Science. v. 21, p.161-168. 2000.

MACEACHREN, A. M. How maps work: Representation, Visualization and Design. London: The Guilford Press, 1995.

MARQUES, A. P. S.; DECANINI, M. M. S.; PUGLIESI, E. A. Sistema de Navegação e Guia de Rota áudio-dinâmico com múltiplas escalas automáticas. Revista Brasileira de Cartografia. v. 1, n. 64, p. 57-68. 2012.

MARTINELLI, M. Mapas da Geografia e Cartografia Temática. 6 ed. Contexto: São Paulo, 2011.

MAY, A. J.; ROSS, T. BAYER, S. H. Drivers' Information Requirements when Navigating in an Urban Environment. The Journal of Navigation. United Kingdom: The Royal Institute of Navigation. v. 56, p. 89-100. 2003.

MICHON, J. A. A critical view of driver behaviour models. In: EVANS, L.; SCHWING, R. S. Human behaviour and traffic safety. University of Groningen, New York: Plenum Press, 1985.

OBATA, T.; DAIMON T.; KAWASHIMA, H. A cognitive study of invehicle navigation systems: aplying verbal protocol analysis to usability evaluation. In: Proceedings of Vehicle Navigation and Information Systems Conference. Piscataway, NJ: Institute of Electrical and Electronics Engineers, p. 232-237. 1993. 
PEDROSA, I. O universo da cor. 4 ed. Senac Nacional: Rio de Janeiro, 2009, 160p. PUGLIESI, E. A.; DECANINI, M. M. S.; IMAI, N. N. HASEGAW, J. K. Projeto cartográfico do mapa dinâmico para um sistema de navegação rodoviário. Revista Brasileira de Cartografia. v. 56, n. 2, p.104-117. 2004.

PUGLIESI, E. A.; DECANINI, M. M. S.; TACHIBANA, V. M. Evaluation of the Cartographic Communication Performance of a Route Guidance and Navigation System. Cartography and Geographic Information Science. v. 36, n. 2, p.193-207. 2009.

PUGLIESI, E. A.; DECANINI, M. M. S.; RAMOS, A. P. M.; TSUCHIYA, I. Métodos para Avaliação da Usabilidade de Sistemas de Navegação e Guia de Rota. Revista Brasileira de Cartografia. v. 5, n. 65, p. 571-589. 2013.

PUGLIESI, E. A.; DECANINI, M. M. S. Mapa ou Esquema de seta: qual modalidade os motoristas preferem para sistemas de navegação e guia de rota em automóvel? Boletim de Ciências Geodésias. v. 15, n. 1, p.03-15. 2009a.

PUGLIESI, E. A.; DECANINI, M. M. S. Projeto cartográfico de sistema de navegação e guia de rota em automóvel com pontos de referência de Alta iconicidade. Revista Brasileira de Cartografia. v. 2, n. 61, p.79-89. $2009 \mathrm{~b}$.

RIGDEN, C. The eye of the beholder - Designing for colour-blind users. British Telecommunications Engineering. v. 17, p. 2-6. 1999.

ROSS, T.; VAUGHAN, G.; BURNETT, G.; MAY, A. Design Guidelines for Route Guidance and Navigation. In: ROSS, T. et al. Human Factors Guidelines for Information Presentation by ATT Systems. Loughborough, UK: HUSAT Research Institute, 1996. Cap. 2.

SEKULER, R.; SEKULER, A. B. Age-Related, Optical Factors and Neural Process. Encyclopedia of Psychology. American Psychological Association/Oxford University Press. v.8, p.180-183. 2000.

SIEGEL, S. Estatística não-paramétrica para as ciências do comportamento. São Paulo: McGraw-Hill, 1975.

SLOCUM, A. T.; MCMASTER, R. B.; KESSLER, F. C.; HOWARD, H. H. Thematic Cartography and Geovisualization. 3nd ed. Prentice Hall, 2009.

WICKENS, C. D.; LEE, J. D.; LIU, Y.; BECKER, S. G. An Introduction to Human Factors Engineering. 2nd ed. California: Pearson Prentice Hall, 2004.

WITTMANN, M.; KISS, M.; GUGG, P.; STEFFEN, A.; FINK, M.; PÖPPEL, E.; KAMIYA, H. Effects of display position of a visual in-vehicle task on simulated driving. Applied Ergonomics. v. 37, p. 187-199. 2006.

WU, A; ZHANG, X. Identifying Proper Scales on Digital Maps for In-Vehicle Navigation Systems. In: Proceedings of HCI, Part III. Springer-Verlag Berlin Heidelberg, p. 262-270. 2009.

YOUNG, M. S.; STANTON, N. A. Applying Interviews to Usability Assessment. In: STANTON, N. A.; et al. The handbook of human factors and ergonomics methods. USA: CRC Press LLC, 2005. Cap. 29. p. 29-1; 29-6.

(Recebido em dezembro de 2013. Aceito em fevereiro de 2014). 